\title{
Dark-Energy Evolution Across the Cosmological-Constant Boundary
}

\author{
Robert R. Caldwell用 and Michael Doran ${ }^{1, \text {, }}$ \\ ${ }^{1}$ Department of Physics \& Astronomy, HB 6127 Wilder Laboratory, Dartmouth College, Hanover, NH 03755, USA \\ ${ }^{2}$ Institut für Theoretische Physik, Philosophenweg 16, 69120 Heidelberg, Germany
}

\begin{abstract}
We explore the properties of dark energy models for which the equation-of-state, $w$, defined as the ratio of pressure to energy density, crosses the cosmological-constant boundary $w=-1$. We adopt an empirical approach, treating the dark energy as an uncoupled fluid or a generalized scalar field. We describe the requirements for a viable model, in terms of the equation-of-state and sound speed. A generalized scalar field cannot safely traverse $w=-1$, although a pair of scalars with $w>-1$ and $w<-1$ will work. A fluid description with a well-defined sound speed can also cross the boundary. Contrary to expectations, such a crossing model does not instantaneously resemble a cosmological constant at the moment $w=-1$ since the density and pressure perturbations do not necessarily vanish. But because a dark energy with $w<-1$ dominates only at very late times, and because the dark energy is not generally prone to gravitational clustering, then crossing the cosmological-constant boundary leaves no distinct imprint.
\end{abstract}

Numerous observations and experiments indicate that our universe has low matter-density, negligible spatial curvature, and is currently undergoing accelerated cosmic expansion [1, 2, 3, 4, 5, 6, 7]. The remarkable implication is that approximately two-thirds of the cosmic energy is due to some form of as-yet unidentified dark energy. While the leading interpretation is that the dark energy is due to a cosmological constant, the physical origin of such a constant remains a mystery, and it is widely regarded as a placeholder until a deeper understanding of the dark energy can be established.

In an effort to characterize the nature of the dark energy, attention has focused on its presumed equation-of-state, $w$, defined as the ratio of its mean pressure to energy density, $w \equiv p / \rho$. A cosmological constant corresponds to $w=-1$. Another conjecture is that the dark energy is due to quintessence, a dynamical, ultra-light scalar field with negative pressure, for which $w>-1$ [8, 9, 10]. A separate class of models with $w<-1$, representing an exotic field or perhaps new gravitational phenomena, are also under investigation (e.g. 11]). Extensive analysis of the cosmological predictions for all these cases finds that the current data favors dark energy models with an equation-of-state in the vicinity of $w=-1$, straddling the cosmological-constant boundary.

If indeed dark energy with $w<-1$ is within the realm of possibilities, then it would seem inevitable to inquire about a transition from $w>-1$ to $w<-1$. This question has been taken up recently 12, 13]; here we contribute our results and perspective on the issue. This article examines possible mechanisms by which dark energy can cross $w=-1$. We assume that Einstein's gravitation is valid and that the dark energy system interacts only gravitationally with the rest of the world - all of our ignorance is captured in $w$. The sticking point is the stability of such a "crossing component," which brings into question the physics of the dark energy.

Let's start from the observations: measurements of luminosity distances based on type 1a supernovae and other phenomena imply a trajectory $a(t)$ for the expansion scale factor in our homogeneous, isotropic universe. General relativity connects this expansion history with the matter and energy sources, in our (approximately) geometrically flat universe, which leads us to infer the existence of a dark energy. Making the simplest assumptions about the nature of this unknown substance, we hypothesize that it can be described as an ideal fluid with a mean energy density and pressure. We can parametrize the dark energy evolution with the present-day abundance $1-\Omega_{m}$ and the equation-of-state trajectory $w(\tau)$ as well as a sound speed for the response of small fluctuations. This procedure is sufficient to allow us to pursue classical tests of cosmology.

A proper comparison of dark energy model predictions with the observed cosmic microwave background anisotropy and galaxy clustering requires that we treat the inhomogeneities in the dark energy. We adopt the conventions and notation of [14], so that we may describe the fluid perturbations according to the conformal-Newtonian gauge equations

$$
\begin{aligned}
& \dot{\delta}=-(1+w)(\theta-3 \dot{\phi})-3 \frac{\dot{a}}{a}(\delta p-w \delta \rho) / \rho \\
& \dot{\theta}=-\frac{\dot{a}}{a}(1-3 w) \theta-\frac{\dot{w}}{1+w} \theta+\frac{\delta p / \delta \rho}{1+w} k^{2} \delta+k^{2}(\psi-\sigma) .
\end{aligned}
$$

\footnotetext{
*Electronic address: Robert.R.Caldwell@Dartmouth.edu
}

${ }^{\dagger}$ Electronic address: M.Doran@gmx.de 
The standard fluid perturbation equations appear to grow singular because the terms proportional to $(1+w)^{-1}$ diverge in the case of a crossing. However, the physically meaningful source of momentum transfer in the standard perturbation equations is $\rho(1+w) \theta$, not $\theta$ alone, so that by defining the fractional momentum density transfer $\mathcal{V} \equiv(1+w) \theta$ we obtain

$$
\begin{aligned}
\dot{\delta} & =-\mathcal{V}+3(1+w) \dot{\phi}-3 \frac{\dot{a}}{a}(\delta p-w \delta \rho) / \rho \\
\dot{\mathcal{V}} & =-\frac{\dot{a}}{a}(1-3 w) \mathcal{V}+k^{2} \delta p / \rho+k^{2}(1+w)(\psi-\sigma)
\end{aligned}
$$

In the synchronous gauge we obtain

$$
\begin{aligned}
\dot{\delta} & =-\mathcal{V}-(1+w) \frac{1}{2} \dot{h}-3 \frac{\dot{a}}{a}(\delta p-w \delta \rho) / \rho \\
\dot{\mathcal{V}} & =-\frac{\dot{a}}{a}(1-3 w) \mathcal{V}+k^{2} \delta p / \rho-k^{2}(1+w) \sigma .
\end{aligned}
$$

With the fluid perturbation equations recast in the above forms, we see that the response of a dark energy density perturbation to an external gravitational field flips sign at crossing. That is, gravitational instability becomes an antigravitational instability. On small scales the effects of shear, which typically damp perturbation growth, are reversed. However, there is nothing in the equations to suggest that the fluid perturbations should vanish at the instant $w=-1$, as we might expect if the dark energy instantaneously looks like a cosmological constant. Without a model of the pressure fluctuations and shear this system of equations is incomplete.

We can close the system of equations and follow the evolution by specifying a relation $\delta p=v^{2}[\tau, k] \delta \rho$ and a function $\sigma[\tau, k]$, but there are many factors to consider. A canonical scalar field has $v^{2}=1$ on small scales; on scales approaching the horizon the relation becomes gauge-dependent. Models of a generalized scalar, such as k-essence [15], can have a variable $v^{2}$. And if $v^{2} \ll 1$ within the horizon then dark energy can cluster [16, 17, 18, 19]. Note that the adiabatic sound speed $\left.c_{s}^{2}\right|_{(\text {adiab. })} \equiv \dot{p} / \dot{\rho}=-d \ln (1+w) / 3 d \ln a$ is a red herring, as it does not actually describe the propagation of spatial inhomogeneities in the dark energy fluid. The inhomogeneous fluctuations generally have more degrees of freedom than the homogeneous background. Of course, whenever $v^{2} \neq\left. c_{s}^{2}\right|_{\text {(adiab.) }}$, the rate of entropy generation $\Gamma=\left(v^{2}-\left.c_{s}^{2}\right|_{(\text {adiab. })}\right) \delta / w$ is non-vanishing. However, let us proceed to construct an admittedly naive, synthetic model with $\sigma=0, v^{2}=1$ on sub-horizon scales to prevent clustering like dark matter, and $v^{2} \rightarrow w$ on super-horizon scales so that the unevolved dark energy perturbations resemble the background. (We are aware of the many myths surrounding super-horizon modes [20].) Our naive model has $v^{2}[\tau, k]=w(a) \exp (-x)+\mu^{2}[1-\exp (-x)]$ applied in the conformal-Newtonian gauge, and where $\mu^{2}=1$ is the small scale speed of sound and $x \equiv k \tau$. It will also be interesting to allow $\mu^{2}=0$. For further variety we propose a second synthetic model with $v^{2}=1$ on all scales, again in the conformal-Newtonian gauge.

The generalized scalar field, employed in k-essence and phantom models, might also serve to describe a dark energy component which crosses $w=-1$. In this scheme, the scalar field Lagrangian originates as a nonlinear function of gradient and field,

$$
S_{d}=\int d^{4} x \sqrt{-g} F(X, \varphi)
$$

Here $X \equiv \frac{1}{2}\left(\partial_{\mu} \varphi\right)\left(\partial^{\mu} \varphi\right)$ and a canonical scalar field is simply given by $F=X+V(\varphi)$. The spatially-uniform energy density and pressure are $\rho=F-2 X F_{, X}$ and $p=-F$. For a field with only linear dependence on $X$, $F=K(\varphi) X+V(\varphi)$ there are two immediate consequences. First, the system can be transformed into a canonical scalar field with positive kinetic energy $(w>-1)$ or negative kinetic energy $(w<-1)$ by a field redefinition, where we note that the equation-of-state is given by $w=F /\left(2 X F_{, X}-F\right)$. Second, such a system leads to well-behaved pressure fluctuations [16, 17, 18],

$$
\delta p=\mu^{2} \delta \rho+\rho k^{-2} \mathcal{V}\left[3 \frac{\dot{a}}{a}\left(\mu^{2}-w\right)+\frac{\dot{w}}{1+w}\right],
$$

where $\mu^{2}=F_{, X} /\left(F_{, X}+2 X F_{, X X}\right)$. If $K$ is a constant, then $\mu^{2}=1$ so that the sound speed reduces to the manifestly stable $v^{2}=1$ on small scales. Relaxing the assumption of a linear dependence of the Lagrangian on $X$, different sound speed histories are possible. But let's see what happens when we push this generalized scalar field across the $w=-1$ boundary.

Consider a dark energy model consisting of a single-component, generalized scalar field. In order to cross at $w=-1$ we require $X=0$ and/or $F_{, X}=0$. One can show that a vanishing $X$ corresponds to an extremum in the equation-ofstate, meaning $w=-1$ is a minimum or maximum. This leaves $F_{, X}=0$ as a necessary condition to cross. However, if 
$F_{, X}$ evolves though zero and $F_{, X X} \neq 0$, then $\mu^{2}$ becomes negative, thereby leading to unstable perturbations. Hence, the path across $w=-1$ would appear to be blocked.

A remaining possibility is to allow for exceptional fine tuning of the scalar field solution. Suppose that we fix $F$ such that $\mu^{2}>0$ for all times. Then the scalar field equation of motion,

$$
\left[\mu^{-2} \ddot{\varphi}+2 \frac{\dot{a}}{a} \dot{\varphi}\right] F_{, X}+a^{2}\left[F_{, \varphi}-2 X F_{, X \varphi}\right]=0,
$$

requires that we somehow tune $F_{, \varphi}-2 X F_{, X \varphi} \rightarrow 0$ just as $F_{, X}$ vanishes. Perhaps a solution can be constructed by working backwards, starting from an assumed evolution for $w(\tau), \mu^{2}(\tau)$. But it should be clear that even a slight shift away from this special trajectory through phase space would prevent a crossing. The consequences of a perturbation at the crossing are still worse, as we now argue, where terms like $\dot{w} /(1+w)$ indeed lead to unphysical behavior. We expand the scalar field relation (5) and the perturbation equations (2), near the crossing time $\tau_{\star}$ in powers of $\tau-\tau_{\star}$. Hence, for a trajectory, $w(\tau)=-1+\alpha\left[\tau-\tau_{\star}\right]^{n}$ where $n$ is an odd integer, we expand the fluid variables

$$
\begin{aligned}
\delta & =\delta^{\star}+\delta^{1}\left[\tau-\tau^{\star}\right]+\ldots \\
\mathcal{V} & =\mathcal{V}^{\star}+\left(\mathcal{V}^{1}+\mathcal{V}^{L} \ln \left|\tau-\tau^{\star}\right|\right)\left[\tau-\tau^{\star}\right]+\ldots
\end{aligned}
$$

The analytic solutions near the crossing give $\mathcal{V}^{\star}=0$, and the next coefficients can be easily obtained. The results show that $\delta\left(\tau_{\star}+\epsilon\right)=\delta\left(\tau_{\star}-\epsilon\right)$ and $\mathcal{V}\left(\tau_{\star}+\epsilon\right)=-\mathcal{V}\left(\tau_{\star}-\epsilon\right)$, for vanishing $\epsilon$. Whereas the velocity perturbation vanishes at crossing, the density perturbation need not. If $n=1$ then the pressure perturbation diverges logarithmically, $\delta p \propto \ln \left|\tau-\tau^{\star}\right|$. If $n \geq 3$ then the perturbations are well-behaved. But something else goes wrong in either case. Look at the expression for the density perturbation: $\delta \rho=\left(F_{, \varphi}-2 X F_{, X \varphi}\right) \delta \varphi-\left(F_{, X}+2 X F_{, X X}\right) \delta X$. If we require $\mu^{2} \neq 0$ at crossing, then the coefficients of both the $\delta \varphi$ and $\delta X$ terms must vanish; in order for the density to be non-zero, one or both of $\delta \varphi$ and $\delta X$ must diverge. If the field and its derivatives are to make any sense, we must abandon this scalar field description as a mechanism for crossing $w=-1$. Hence, there is no viable path for the scalar field across the cosmological-constant boundary. These findings are consistent with the thorough analysis of Vikman [12].

If the scalar field itself cannot cross $w=-1$, then there is a simple way to cross with two fields, as pointed out by $\mathrm{Hu}$ [13]. Consider one scalar field with equation-of-state $w_{1}>-1$ and a second, generalized scalar with $w_{2}<-1$ : together these can be used to describe a fluid with energy density $\rho_{12}=\rho_{1}+\rho_{2}$ dominated by the first field at early times and the second field at late times. And since both components yield stable fluctuations, the ensemble is also stable. Then, suppose we have a trajectory $w_{12}(a)$ which describes such a dark energy that crosses at $a=a_{\star}$. There is not a unique prescription to break this into two components, but we can be economical by requiring that $w_{1} \equiv \bar{w}_{1}=$ constant for $a>a_{\star}$ and $w_{2}=\bar{w}_{2}=$ constant for $a<a_{\star}$. Since $\rho_{12}(a)=\rho_{12}\left(a_{0}\right) \exp \left(3 \int_{a}^{a_{0}}\left(1+w_{12}\right) d \ln a\right)$, we can use energy conservation and the continuity of the energy density to show that

$$
\begin{aligned}
w_{1}\left(a<a_{\star}\right) & =\bar{w}_{2}+\left(w_{12}-\bar{w}_{2}\right) /\left[1-\frac{1+\bar{w}_{1}}{\bar{w}_{1}-\bar{w}_{2}} \frac{\rho_{12}^{\star}}{\rho_{12}}\left(\frac{a_{\star}}{a}\right)^{3\left(1+\bar{w}_{2}\right)}\right] \\
w_{2}\left(a>a_{\star}\right) & =\bar{w}_{1}+\left(w_{12}-\bar{w}_{1}\right) /\left[1-\frac{1+\bar{w}_{2}}{\bar{w}_{2}-\bar{w}_{1}} \frac{\rho_{12}^{\star}}{\rho_{12}}\left(\frac{a_{\star}}{a}\right)^{3\left(1+\bar{w}_{1}\right)}\right] \\
f & =\frac{\left(1+\bar{w}_{2}\right)}{\bar{w}_{2}-\bar{w}_{1}} \frac{\rho_{12}^{\star}}{\rho_{12}^{0}}\left(\frac{a_{\star}}{a_{0}}\right)^{3\left(1+\bar{w}_{1}\right)} .
\end{aligned}
$$

This last expression also gives the relative abundance of the first component at the present day, $f=\rho_{1}^{0} / \rho_{12}^{0}$. We have to be somewhat careful with this piecewise construction, since $\dot{w}_{1,2}$ is discontinuous at the crossing. If we choose $\bar{w}_{1,2}$ to be very close to -1 , then immediately before/after $a_{\star}$ the ratio $\dot{w}_{1,2} /\left(1+w_{1,2}\right)$ which appears in (5) will be very large. Alternatively, two smooth equation-of-state histories $w_{1}(a) \geq-1$ and $w_{2}(a) \leq-1$ and a relative abundance $f$ can be chosen to give a composite, scalar-field crossing model.

To examine the observational consequences of a crossing dark energy component, we consider two toy-model scenarios for $w(a)$. For the first (EOS I) we take $w(a)=-3 / 2+(1-a)$, and for the second (EOS II) we use $w(a)=-1-\tanh \left(10\left[a-\frac{1}{2}\right]\right)$. Both are shown in Figure 1 Fixing $\Omega_{m}=0.3$ today, then we find that EOS I leads to negligible dark energy at early times whereas EOS II for which $w \rightarrow 0$ at early times, contributes a non-negligible fraction of the total energy density throughout the matter-dominated era. Reducing the factor in the tanh from 10, however, greatly reduces the abundance of dark energy in the matte rera. We also investigated a third toy-model, consisting of a pair of scalar fields with $w_{1}(a) \geq-1, w_{2}(a) \leq-1$ which are smoothly-varying; these components were contrived to produce the ensemble evolution of EOS II. However, we found no relevant differences with the piecewise construction.

We modified CMBeasy 21] to study the consequences of these models. In neither case is there a discernable trace of the mechanism used to achieve a cosmological-constant crossing. The synthetic fluid and the two-scalar 
approach produce essentially identical results. (We use the log-likelihood statistic introduced in Ref. 22] to check for degeneracies.) This holds whether $\mu^{2}=1$ or $v^{2}=1$ on all scales. There is a distinction between models with $\mu^{2}=0$ and $\mu^{2}=1$, but this is independent of the dark energy composition. Rather, perturbations in model EOS II with $\mu^{2}=0$ grow from the initial, adiabatic conditions throughout the matter-dominated era during which time the dark energy has $w=0$, too; in the present era, the dark energy comes to dominate, $w$ crosses -1 , and the growth is not only slowed but reversed as the dark energy lumps respond anti-gravitationally to the gravitational potentials. The observational imprint is an additional ISW contribution and boost to the mass power spectrum, both typically on the order of $\sim 10 \%$ for the cases we have considered. Reducing the factor in the tanh from 10 greatly reduces the impact of any additional clustering.

The evolution of dark energy density perturbations in these models is of some interest. For model EOS I the perturbations never grow large; the negative equation-of-state and negligible energy density until very late times keep the fluctuations from making a significant impact. (To neglect the perturbations completely, however, is equivalent to a violation of energy conservation. The degree to which this influences theoretical predictions depends on the gauge in which the fluctuations are ignored.) For EOS II the perturbations grow significantly in the $\mu^{2}=0$ case. Starting from adiabatic initial conditions, the density perturbations grow like $\delta \propto a$ through the matter-dominated era. As illustrated in Figure 2 when the dark energy comes to dominate, the growth rate slows and eventually the density contrast is driven negative. In contrast, the $v^{2}, \mu^{2}=1$ models suppress fluctuation growth. We also notice that the density contrast for high-frequency modes vanishes when $w$ crosses - 1, as seen in Figure 2 For these high frequency modes the oscillations have been long suppressed by the expansion, and the density directly tracks the driving term, which is proportional to $(1+w)$. When the sources vanish, so do the dark energy fluctuations. Only in this instant does the dark energy instantaneously resemble a smooth, cosmological constant.

We have now described several simple ways to engineer a $w=-1$ crossing. The primary tool for modeling dark energy is the scalar field, which must possess an unorthodox, negative kinetic term to achieve $w<-1$. There are substantial reasons to find such a field objectionable [23], although we are willing to keep such possibilities open until observations and experiment give us a better idea as to the nature of the dark energy.

Other mechanisms have been proposed to explain a dark energy $w=-1$ crossing. First, a dark matter-dark energy interaction which siphons energy from CDM into a quintessence field can produce an expansion history which appears as though the cosmos is dominated by a non-interacting CDM and a crossing dark energy [24, 25, 26]. However, such a coupling may be difficult to realize because of quantum effects [27]. Likewise, scalar-tensor theories can mimic a crossing under less-extreme circumstances than a negative-kinetic cosmic scalar field. Second, a cosmic field which undergoes a burst of particle production has been suggested as a means to produce pole-like inflation. Transplanting this mechanism from early- to late-times, the field may be used to drive super-acceleration without requiring a super-negative pressure [28, 29]. Third, higher-order or non-perturbative quantum effects as occur in the vacuum metamorphosis model can cause an otherwise well-behaved scalar field to push the cosmos across the $w=-1$ boundary [30]. In these scenarios there are other effects - variations in particle masses or coupling constants, features in the CMB anisotropy and mass power spectra - that may be exploited to identify the underlying mechanism.

If observational and experimental evidence grows sufficiently compelling to favor a component that has $w<-1$ for some duration, and if conventional or astrophysical effects cannot account for the observed phenomena, then it may be necessary to consider the cosmological-constant boundary-crossing scenarios.

\section{Acknowledgments}

This work was supported in part by NSF AST-0349213 at Dartmouth and DFG grant 1056/6-3 at Heidelberg.

[1] A. G. Riess et al. [Supernova Search Team Collaboration], Astrophys. J. 607, 665 (2004).

[2] D. N. Spergel et al. [WMAP Collaboration], Astrophys. J. Suppl. 148, 175 (2003).

[3] A. C. S. Readhead et al., Astrophys. J. 609, 498 (2004).

[4] J. H. Goldstein et al., Astrophys. J. 599, 773 (2003) arXiv:astro-ph/0212517.

[5] R. Rebolo et al., arXiv:astro-ph/0402466

[6] M. Tegmark et al. [SDSS Collaboration], Phys. Rev. D 69, 103501 (2004).

[7] E. Hawkins et al., Mon. Not. Roy. Astron. Soc. 346, 78 (2003).

[8] C. Wetterich, Nucl. Phys. B 302, 668 (1988).

[9] B. Ratra and P. J. Peebles, Phys. Rev. D 37, 3406 (1988).

[10] R. R. Caldwell, R. Dave and P. J. Steinhardt, Phys. Rev. Lett. 80, 1582 (1998).

[11] R. R. Caldwell, Phys. Lett. B 545, 23 (2002). 
[12] A. Vikman, arXiv:astro-ph/0407107

[13] W. Hu, arXiv:astro-ph/0410680

[14] C. P. Ma and E. Bertschinger, Astrophys. J. 455 7, (1995).

[15] C. Armendariz-Picon, V. Mukhanov and P. J. Steinhardt, Phys. Rev. D 63, 103510 (2001).

[16] J. K. Erickson, R. R. Caldwell, P. J. Steinhardt, C. Armendariz-Picon and V. Mukhanov, Phys. Rev. Lett. 88, 121301 (2002).

[17] S. DeDeo, R. R. Caldwell and P. J. Steinhardt, Phys. Rev. D 67, 103509 (2003); [Erratum-ibid. D 69, 129902 (2004)].

[18] R. Bean and O. Dore, Phys. Rev. D 69, 083503 (2004).

[19] J. Weller and A. M. Lewis, Mon. Not. Roy. Astron. Soc. 346, 987 (2003).

[20] W. H. Press and E. T. Vishniac, Astrophys. J. 239, 1 (1980).

[21] M. Doran, arXiv:astro-ph/0302138

[22] G. Huey, L. M. Wang, R. Dave, R. R. Caldwell and P. J. Steinhardt, Phys. Rev. D 59, 063005 (1999).

[23] S. M. Carroll, M. Hoffman and M. Trodden, Phys. Rev. D 68, 023509 (2003).

[24] C. Wetterich, Astron. Astrophys. 301, 321 (1995).

[25] L. Amendola, Phys. Rev. D 62, 043511 (2000).

[26] G. Huey and B. D. Wandelt, arXiv:astro-ph/0407196

[27] M. Doran and J. Jaeckel, Phys. Rev. D 66, 043519 (2002).

[28] J. D. Barrow, Class. Quant. Grav. 21, L79 (2004).

[29] W. Zimdahl, D. J. Schwarz, A. B. Balakin and D. Pavon, Phys. Rev. D 64, 063501 (2001).

[30] L. Parker and A. Raval, Phys. Rev. D 60, 063512 (1999); [Erratum-ibid. D 67 (2003) 029901]. 


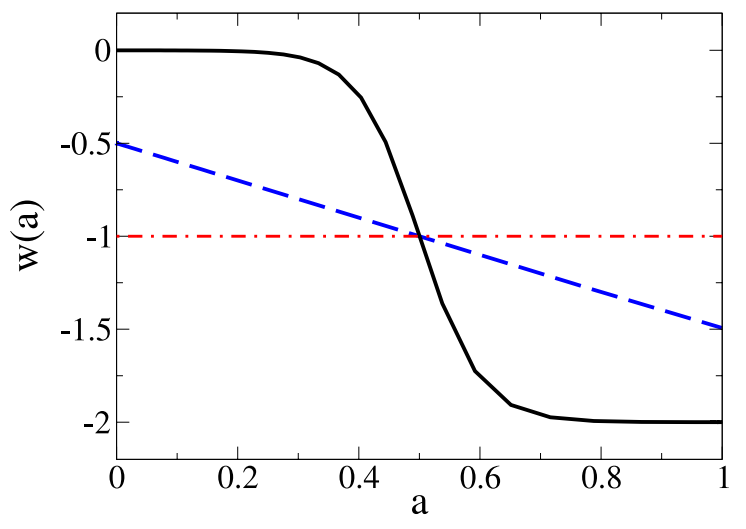

FIG. 1: The equation-of-state $w(a)$ for two toy models which cross the cosmological-constant boundary are shown. EOS I is the dashed line, and EOS II is the solid curve. In both cases, dark energy evolves into the phantom regime, below the dot-dashed line, beginning from $a_{\star}=1 / 2$. For EOS II the dark energy evolves as matter at early times, since $w \rightarrow 0$, and contributes measurably to the energy budget of the Universe throughout matter domination.

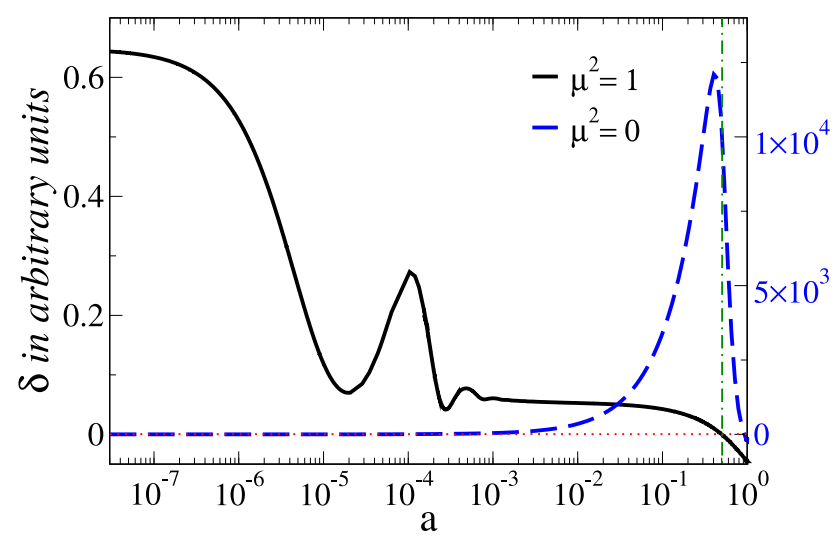

FIG. 2: The density contrast for a pair of high-frequency modes with $\mu^{2}=1$ (solid curve) and $\mu^{2}=0$ (dashed) for the EOS II synthetic fluid. As explained in the text, the density contrast for the $\mu^{2}=1$ case vanishes when $w=-1$, instantaneously resembling a cosmological constant with no fluctuations. In both cases the density contrast eventually grows negative, as the gravitational instability flips to an antigravitational instability. (Note the left scale is for the solid curve; the right scale is for the dashed curve.) 